\title{
STABILITY OF RETRIAL QUEUES WITH VERSATILE RETRIAL POLICY
}

\author{
TEWFIK KERNANE AND AMAR AÏSSANI
}

Received 19 December 2004; Revised 22 November 2005; Accepted 22 November 2005

We consider in this paper the stability of retrial queues with a versatile retrial policy. We obtain sufficient conditions for the stability by the strong coupling convergence to a stationary ergodic regime for various models of retrial queues including a model with two types of customers, a model with breakdowns of the server, a model with negative customers, and a model with batch arrivals. For all the models considered we assume that the service times are general stationary ergodic and interarrival and retrial times are i.i.d. sequences exponentially distributed. For the model with unreliable server we also assume that the repair times are stationary and ergodic and the occurrences of breakdowns follow a Poisson process.

Copyright (C 2006 T. Kernane and A. Aïssani. This is an open access article distributed under the Creative Commons Attribution License, which permits unrestricted use, distribution, and reproduction in any medium, provided the original work is properly cited.

\section{Introduction}

We investigate in this paper the problem of stability condition for some retrial queueing models. The first model is a retrial queue with a versatile retrial policy which incorporates simultaneously the classical linear retrial policy and the constant one and is described as follows. Consider a single server queue in which a primary arriving customer who finds the server busy moves to a group of blocked customers called "orbit" and repeatedly retries for service until he finds the server free, and consider the following retrial policy for the access to the server from the orbit. The probability of a repeated attempt from the orbit during the interval $(t, t+\Delta t)$, given that $j$ customers were in orbit at time $t$, is $\left(\theta\left(1-\delta_{0 j}\right)+j \mu\right) \Delta t+\circ(\Delta t)$. This model was defined by Artalejo and Gomez-Corral [4]. If $\mu=0$, we obtain the constant retrial policy with parameter $\theta$ introduced by Fayolle [14]. If a primary arriving customer finds the server free, he receives service and leaves the system. The second model is a retrial queue with two types of arriving customers, known as "impatient" and "persistent." If an impatient customer finds the server busy, then it leaves the system. On the other hand, if a persistent customer arrives and finds the server busy, then he may have access to the orbit and waits to be served later according to the 
versatile retrial policy described above. If $\mu=0$, we obtain the model with constant retrial policy and two types of customers studied by Martin and Artalejo [21]. The third model is a single server retrial queue with the server subject to random breakdowns and repairs. In the fourth model we consider the stability of a retrial queue with positive and negative customers. When a negative arrival occurs in the system, it immediately removes one regular customer if present. The concept of negative customers was introduced by Gelenbe [17], who established the product form solution for a queueing network including negative arrivals as well as regular ones. A review of results and practical situations can be found in Artalejo [3]. Gelenbe et al. [18] derived the stability conditions for two models of negative arrivals, the removal of customer in service (RCS) and removal of customer at the tail of the queue (RCT). Artalejo and Gomez-Corral $[5,6]$ extended the queues with negative arrivals to that when regular customers follow a retrial policy. Finally, we consider the stability of a retrial queue with batch arrivals. The first such model with the classical linear retrial policy was introduced by Falin [12], who derived the joint distribution of the server state and queue length. A more detailed analysis of the model was given later by Falin [13].

Most of the efforts in the study of retrial queues have been directed to the computation of the steady-state solutions assuming that such stationary regime exists. For an $\mathrm{M} / \mathrm{G} / 1$ queue with linear retrial policy under Markovian assumptions, that is, Poisson arrivals with rate $\lambda$, i.i.d. exponential retrial times with rate $\theta$, and i.i.d. general service times with rate $\mu$, the necessary and sufficient condition for stability is $\lambda<\mu$. Intuitively, we would expect that this condition is indeed sufficient in general. But, Liang and Kulkarni [20] gave a counterexample showing that the system can be unstable even though the arrival rate is less than the service rate. The problem of deriving stability condition becomes nontrivial also when perturbing independence and/or exponential assumptions upon parametric distributions, it is essentially due to the difficulty of describing the basic process in terms of a Markov chain in a simple way. Similar arguments can be provided in the case of the model with constant retrial policy in which case the stability condition depends in general on the retrial rate.

We consider in this paper the approach based on constructing a class of processes, the so-called stochastic recursive sequences, which are of a more general and complicated nature than Markov processes and using the techniques of renovation events to obtain strong coupling convergence to a stationary ergodic regime. These techniques were formulated, in the stationary ergodic context, by Borovkov [9]. The approach of renovating events already has its roots in the early work by Akhmarov and Leont'eva [1]. These methods have further been developed in [7, 15, 16]. For the classical linear retrial queue, Altman and Borovkov [2] obtained sufficient conditions for the stability under various general assumptions for interarrival and service times, in particular they applied the method of renovation events to obtain ergodicity under general stationary ergodic service times and independent and exponentially distributed interarrival and retrial times. In Section 2, we give some backgrounds for stochastic recursive sequence theory and review the main theorem that enables us to obtain a strong coupling convergence to a stationary regime by using renovation events for the retrial queues described above. In Section 3, we apply the method of stochastic recursive sequences to the retrial queue 
with the versatile retrial policy and we derive a sufficient stability condition under general assumption of stationary and ergodic service times, exponentially distributed interarrival and retrial times. In Section 4, we derive a sufficient stability condition for the retrial queue with two types of customers under general stationary ergodic service times for both types, and exponentially distributed interarrival and retrial times. In Section 5, we use the same method for the retrial queue with breakdowns and repairs to derive a sufficient stability condition under the assumptions that the service and repair times are stationary and ergodic sequences and independent and exponentially distributed interarrival, breakdown, and retrial times. In Section 6, we obtain a sufficient stability condition for the retrial queue with negative customers by assuming that the service times of the regular customers are stationary and ergodic, interarrival times of regular and negative customers and retrial times are independent and exponentially distributed. In Section 7, we consider a retrial queue with the versatile retrial policy in which customers arrive in batches and we derive a sufficient condition for stability under the assumptions that the service times are stationary and ergodic, interarrival and retrial times are independent and exponentially distributed and the sizes of batches of arrivals are independent and generally distributed.

\section{Stochastically recursive sequences}

Let $X=\{X(n), n \geq 0\}$ and $\left\{\xi_{n}\right\}$ be random sequences defined on the same probability space $(\Omega, \mathfrak{I}, P)$ and taking values in measurable spaces $\left(\mathbf{X}, B_{\mathbf{X}}\right)$ and $\left(\mathbf{Y}, B_{\mathbf{Y}}\right)$, respectively. Assume moreover, that a measurable function $f: \mathbf{X} \times \mathbf{Y} \rightarrow \mathbf{X}$ is defined on $\left(\mathbf{X} \times \mathbf{Y}, B_{\mathbf{X}} \times\right.$ $\left.B_{\mathrm{Y}}\right)$.

Definition 2.1. A random sequence $\{X(n)\}$ is called a stochastically recursive sequence (SRS) controlled by the governing sequence $\left\{\xi_{n}\right\}$ if $\{X(n)\}$ obey the equation

$$
X(n+1)=f\left(X(n), \xi_{n}\right), \quad \forall n \geq 0
$$

Stochastically recursive sequences (SRS) are more general objects than Markov chains, that is, each Markov chain can be represented as an SRS with independent $\xi_{n}$ (see Kifer [19] or Borovkov [10]).

We assume in the sequel that the sequence $\left\{\xi_{n}\right\}$ is stationary (in the strict sense), that is, the distributions of the finite-dimensional random variables $\left(\xi_{k+n_{1}}, \xi_{k+n_{2}}, \ldots, \xi_{k+n_{j}}\right)$ do not depend on $k$ for any $j$ and $n_{1}, \ldots, n_{j}$. By the Kolmogorov's theorem on the extension of compatible distributions, a stationary sequence $\left\{\xi_{n} ; n \geq 0\right\}$ may be extended to obtain the sequence $\left\{\xi_{n} ;-\infty<n<\infty\right\}$ stationary on the entire time-axis. Let us introduce the $\sigma$-algebras $\mathfrak{J}_{n}^{\xi}=\sigma\left(\xi_{k} ; k \leq n\right)$ and $\mathfrak{J}^{\xi}=\sigma\left(\xi_{k} ;-\infty<k<\infty\right)$.

Definition 2.2. An event $A \in \mathfrak{J}_{n+m}^{\xi}, m \geq 0$, is a renovation event for the SRS $X(n)$ on the segment $[n, n+m]$ if there exists a measurable function $g: \mathbf{Y}^{m+1} \rightarrow \mathbf{X}$ such that on the set $A$,

$$
X(n+m+1)=g\left(\xi_{n}, \ldots, \xi_{n+m}\right) .
$$


4 Stability of retrial queues with versatile retrial policy

The sequence $A_{n}, A_{n} \in \mathfrak{J}_{n+m}^{\xi}$, is a renovating sequence of events for the SRS $X(n)$ if there exists an integer $n_{0}$ such that (2.2) holds true for $n \geq n_{0}$ with a common function $g$ for all $n$. Let $U$ be the measure-preserving shift transformation of $\mathfrak{J}^{\xi}$-measurable random variables generated by $\xi_{n}, U \xi_{n}=\xi_{n+1}$, and $U^{k}, k \geq 0$, denote the $k$ th iteration of $U$. A sequence $\left\{\eta_{n} ;-\infty<n<\infty\right\}$ is said to be compatible with the shift $U$ if for any $n \in \mathbb{Z}, \eta_{n}$ is $\mathfrak{J}^{\xi}$-measurable and $U \eta_{n}=\eta_{n+1}$. We denote by $T$ the corresponding transformation of events in the $\sigma$-algebra $\mathfrak{J}^{\xi}$ :

$$
T\left\{\omega: \xi_{j}(\omega) \in B_{j} ; j=1, \ldots, k\right\}=\left\{\omega: \xi_{j+1}(\omega) \in B_{j} ; j=1, \ldots, k\right\}
$$

and $T^{k}, k \geq 0$, denote the $k$ th iteration of $T . U^{0}$ and $T^{0}$ are identity transformations, and $U^{-k}, T^{-k}$ are the inverse transformations for $U^{k}$ and $T^{k}$, respectively.

Remark 2.3. The $\sigma$-algebra $\mathfrak{J}^{\xi}$ may be considered as a possibly larger $\sigma$-algebra in which an arbitrary sequence of independent random variables $\left\{\zeta_{n}\right\}$, taking values in a measurable space $\left(Z, B_{\mathbf{Z}}\right)$, and not depending on $\left\{\xi_{n}\right\}$, is $\mathfrak{J}^{\xi}$-measurable since in this case $\mathfrak{J}^{\xi}$ can be viewed as the $\sigma$-algebra generated by $\left\{\xi_{n}\right\}$ and $\left\{\zeta_{n}\right\}$ (we can denote it by $\mathfrak{J}^{\xi, \zeta}$ ). The shift $T$ will also be defined by means of the relation

$$
\begin{aligned}
T\{\omega: & \left.\left(\xi_{n+i}(\omega), \zeta_{n+i}(\omega)\right) \in B_{i} ; i=0, \ldots, k\right\} \\
& =\left\{\omega:\left(\xi_{n+i+1}(\omega), \zeta_{n+i+1}(\omega)\right) \in B_{i} ; i=0, \ldots, k\right\},
\end{aligned}
$$

for any sets $B_{1}, \ldots, B_{k} \in B_{\mathrm{Y}} \times B_{\mathrm{Z}}$ (see Borovkov [8, page 14]). Let $U$ be the corresponding measure-preserving shift transformation of $\mathfrak{J}^{\xi}, \zeta$-measurable random variables, then for any $\mathfrak{J}^{\xi}, \zeta$-measurable random variable $\eta$ the sequence $\left\{\eta_{n}=U^{n} \eta,-\infty<n<\infty\right\}$ is obviously stationary (see Doob [11, page 452]), and consequently any sequence compatible with the shift $U$ is stationary.

A set $A \in \mathfrak{J}^{\xi}$ is called invariant with respect to the shift $T$ if $A=T A$ almost surely.

A sequence $\left\{\xi_{n}\right\}$ is called metrically transitive if the only invariant sets are those which have probability 0 or 1 .

A sequence $\left\{\xi_{n}\right\}$ is ergodic if and only if for an arbitrary $\mathfrak{J}^{\xi}$-measurable random variable $\eta$, with $\mathrm{E} \eta<\infty$, we have almost surely

$$
\lim _{n \rightarrow \infty} \frac{1}{n} \sum_{i=1}^{n} U^{i} \eta=\mathbf{E} \eta .
$$

If the sequence $\left\{\xi_{n}\right\}$ is stationary, the relation (2.5) can be expressed in the following form:

$$
\lim _{n \rightarrow \infty} \frac{1}{n} \sum_{i=-n}^{-1} U^{i} \eta=\mathbf{E} \eta
$$

The later is called the Birkhoff strong law of large numbers. 
A stationary sequence $\left\{\xi_{n}\right\}$ is ergodic if and only if it is metrically transitive (see Doob [11, Chapter 10]).

Remark 2.4. If $\left\{\xi_{n}\right\}$ is stationary and ergodic, then any sequence $\left\{\eta_{n} ;-\infty<n<\infty\right\}$ compatible with the shift $U$ is also ergodic, that is, it satisfies the Birkhoff strong law of large numbers (see Doob [11, pages 455-456]).

We call the sequence of events $\left\{A_{n}\right\}$ stationary if $A_{k}=T^{k} A_{0}$ for all $k$. Let $X^{0}$ be a random variable with values in the space $\mathbf{X}$ that is measurable with respect to the $\sigma$ algebra $\mathfrak{J}^{\xi}$. Let $\left\{X^{n}=U^{n} X^{0}\right\}$ be the stationary sequence constructed using $X^{0}$.

Definition 2.5. An SRS $\{X(n)\}$ coupling converges to $\left\{X^{n}\right\}$ if it satisfies

$$
\lim _{n \rightarrow \infty} P\left\{X(k)=X^{k}, \forall k \geq n\right\}=1 .
$$

If we introduce the random variable

$$
\nu_{0} \equiv \min \left\{n \geq 0: X(k)=X^{k}, \forall k \geq n\right\}
$$

the relation (2.7) becomes equivalent to

$$
P\left(\nu_{0}<\infty\right)=1
$$

Put

$$
\begin{gathered}
X_{k}(n)=U^{-k} X(n+k), \quad \text { for } n \geq-k, \\
v_{k}=\min \left\{n \geq-k: X_{k}(n)=X^{n}\right\} .
\end{gathered}
$$

Denote by $v=\sup _{k \geq 0} \nu_{k}$ the time when all the sequences $\left\{X_{k}(n), n \geq 0\right\}, k \geq 0$, have fused with the sequence $\left\{X^{n}\right\}$.

Definition 2.6. A sequence $\{X(n)\}$ is strong coupling convergent to the sequence $\left\{X^{n} \equiv\right.$ $\left.U^{n} X^{0}\right\}$ if

$$
\nu<\infty \quad \text { a.s. }
$$

This $v$ is called the strong coupling time. Note that strong coupling convergence is stronger than coupling convergence, which implies the convergence in total variation and then the convergence in distribution. The following theorem (of Borovkov [10, Theorem 11.4]) gives a necessary and sufficient condition of strong coupling convergence of an SRS to a stationary ergodic process.

THEOREM 2.7. The existence of a stationary sequence of renovation events $A_{n}$ with $P\left(A_{n}\right)>0$ is the necessary and sufficient condition of strong coupling convergence of the SRS $X(n)$ to a stationary sequence $X^{n}$ obeying the equation $X^{n+1}=f\left(X^{n}, \xi_{n}\right)$ where $\xi_{n}$ is stationary and ergodic. 


\section{Stability of the retrial queue with versatile retrial policy}

We consider a single server retrial queue at which primary customers arrive to the system at times $\left\{t_{i}, i=1,2, \ldots\right\}$. Let $\tau_{i}=t_{i+1}-t_{i}$ be the successive interarrival times, $i=1,2, \ldots$. If the $i$ th arriving customer finds the server free, he receives service and leaves the system. Otherwise, if the server is busy, the arriving customer moves immediately to an "orbit." The probability of a repeated attempt from the orbit during the interval $(t, t+\Delta t)$, given that $j$ customers were in orbit at time $t$, is $\left(\theta\left(1-\delta_{0 j}\right)+j \mu\right) \Delta t+\circ(\Delta t)$. That is, after an exponential random time with rate $\theta$ (which we will call the orbit retrial time), independent of the arrival process, each customer in orbit generates a Poisson stream of repeated attempts with parameter $\mu$ and behaves independently of other customers in orbit and of the external arrival process. This model, introduced by Artalejo and Gomez-Corral [4], incorporates simultaneously the classical linear retrial policy and the constant one. If $\mu=0$, we obtain the constant retrial policy with parameter $\theta$; if the orbit retrial time ends before an external arrival, then one customer from the orbit (the customer at the head of the queue or a randomly chosen one) occupies the server. The $n$th service duration is $\sigma_{n}$, and we assume that $0<\mathrm{E} \sigma_{n}<\infty$. We assume throughout this section that the sequence $\left\{\sigma_{n}\right\}$ is stationary and ergodic, the interarrival times $\left\{\tau_{i}\right\}$ are i.i.d. exponentially distributed with parameter $\lambda$, the interarrival times, orbit retrial times, and retrial times of each customer in orbit are mutually independent and independent of the sequence $\left\{\sigma_{n}\right\}$. Let $X(t)$ be the number of customers in orbit at time $t$. Define $s_{n}$ to be the instant when the $(n-1)$ st service time ends. We consider the process $X(n)$ embedded immediately after time $s_{n}$, (i.e., $X(n)=X\left(s_{n}^{+}\right)$). After the end of the $(n-1)$ st service, a competition between two independent (since the orbit retrial time and retrial times of each customer in orbit are independent of the interarrival time) exponential laws with rates $\lambda$ and $\theta+X(n) \mu$ determines the next customer that gains the server and the probability that a retrial time expires earlier than the interarrival time is $(\theta+X(n) \mu) /(\lambda+\theta+X(n) \mu)$. Let $u_{n}^{1}$ and $u_{n}^{2}$ be two i.i.d. sequences of random variables distributed uniformly on $[0,1]$, mutually independent and independent of the sequence $\sigma_{n} . u^{1}=\left\{u_{n}^{1}\right\}$ will generate the arrival process, and $u^{2}=\left\{u_{n}^{2}\right\}$ will generate the type of arrival (external or from the orbit) at the end of the successive service periods. Let $\prod: \mathbb{R}_{+} \times[0,1] \rightarrow \mathbb{N}$ denote the inverse of the Poisson distribution

$$
\prod(t, x)=\inf \left\{n \in \mathbb{N}: \sum_{k=0}^{n} \frac{t^{k} e^{-t}}{k !} \geq x\right\},
$$

so that $\prod\left(t, u_{n}^{1}\right)$ is a Poisson random variable with parameter $t$. We have the following representation of the process $X(n)$ :

$$
X(n+1)=\left(X(n)+\xi_{n}\right)^{+}
$$

where $x^{+}=\max [0, x]$ and

$$
\xi_{n}=\prod\left(\lambda \sigma_{n}, u_{n}^{1}\right)-I\left(u_{n}^{2} \leq \frac{\theta+X(n) \mu}{\lambda+\theta+X(n) \mu}\right)
$$

is the governing sequence. 
THEOREM 3.1. The process $X(n)$ is strong coupling convergent to a unique stationary ergodic regime if one of the following conditions is fulfilled:

(1) $\theta>0, \mu=0$, and $\lambda \mathbf{E} \sigma_{1}<\theta /(\lambda+\theta)$;

(2) $\theta \geq 0, \mu>0$, and $\lambda \mathrm{E} \sigma_{1}<1$.

Proof. Consider the case $\theta>0$ and $\mu=0$, then the driving sequence (3.3) has the form

$$
\xi_{n}=\prod\left(\lambda \sigma_{n}, u_{n}^{1}\right)-I\left(u_{n}^{2} \leq \frac{\theta}{\lambda+\theta}\right)
$$

Since the sequence $\left\{u_{n}^{i}\right\}$ is identically distributed for $i=1,2$, and hence stationary, it can be defined for all integers $-\infty<n<\infty$. Define the $\sigma$-algebras $\mathfrak{J}_{n}^{\sigma, u}=\sigma\left(\sigma_{k}, u_{k}^{1}, u_{k}^{2} ; k \leq n\right)$ and $\mathfrak{J}^{\sigma, u}=\sigma\left(\sigma_{k}, u_{k}^{1}, u_{k}^{2} ;-\infty<k<\infty\right)$. Let $U$ be the measure-preserving shift transformation of $\mathfrak{J}^{\sigma, u}$-measurable random variables generated by $\left\{\sigma_{n}, u_{n}^{1}, u_{n}^{2} ;-\infty<n<\infty\right\}$. From Remark 2.3 , since for any $n \in \mathbb{Z}$, the random variable $\xi_{n}$ is generated by $\left\{\sigma_{n}, u_{n}^{1}, u_{n}^{2}\right\}$, then $\xi_{n+1}=U \xi_{n}$ and $\left\{\xi_{n} ;-\infty<n<\infty\right\}$ is stationary. Moreover, since $\left\{\sigma_{n} ;-\infty<n<\infty\right\}$ is stationary and ergodic and the sequence $\left\{\xi_{n} ;-\infty<n<\infty\right\}$ is compatible with the shift $U$, then by Remark 2.4 the sequence $\left\{\xi_{n} ;-\infty<n<\infty\right\}$ is ergodic. We have

$$
\mathbf{E}\left(\xi_{n}\right)=\lambda \mathbf{E} \sigma_{1}-\frac{\theta}{\lambda+\theta}
$$

so that if

$$
\lambda \mathbf{E} \sigma_{1}<\frac{\theta}{\lambda+\theta}
$$

holds, then $\mathrm{E}\left(\xi_{n}\right)<0$. With no loss of generality, we assume that $X(0)=a \geq 0$. Whatever the choice of $n_{0}$, the events $A_{n}=T^{n} A_{0}$, where

$$
A_{0}=\bigcap_{k=0}^{n_{0}-1}\left\{\xi_{-1}+\cdots+\xi_{-1-k} \leq 0\right\} \bigcap_{l \geq 1}\left\{\xi_{-1}+\cdots+\xi_{-n_{0}-l} \leq-a\right\},
$$

make a stationary sequence of renovation events with $m=0$ and $g(y) \equiv y^{+}$(see Borovkov [10, Example 11.1]). Indeed, for $n \geq n_{0}$,

$$
X(n+1)=\xi_{n}^{+} \quad \text { a.s. on } A_{n}
$$

Since $\mathbf{E}\left(\xi_{n}\right)<0$ and the sequence $\left\{\xi_{n}\right\}$ is stationary and ergodic, then by the Birkhoff strong law of large numbers for ergodic sequences, we have almost surely

$$
\lim _{n \rightarrow \infty} \frac{1}{n} \sum_{i=-n}^{-1} \xi_{i}=\mathbf{E} \xi_{1}<0
$$

and it follows that almost surely

$$
\lim _{n \rightarrow \infty}\left(\xi_{-1}+\cdots+\xi_{-n}\right)=-\infty
$$


hence there is a number $n_{0}=n_{0}(a)$ such that $P\left(A_{n}\right)>0$ for $n \geq n_{0}$. If, on the other hand, the events $B_{n}$, the number $m$, and the function $g: \mathbb{R}^{m+1} \rightarrow \mathbb{R}$ are defined as

$$
m=n_{0}, \quad B_{n}=T^{m} A_{n}, \quad g\left(y_{0}, \ldots, y_{m}\right) \equiv y_{m}^{+},
$$

then the events $B_{n} \in \mathfrak{J}_{n+m}^{\xi}$ are renovating for $\{X(n)\}$ on the segment $[n, n+m]$ for all $n \geq 0$, so one can assume that $n_{0}=0$. Hence, using Theorem 2.7, the sequence $\{X(n)\}$ is strong coupling convergent to a unique stationary sequence $\left\{X^{n} \equiv U^{n} X^{0}\right\}$, where $X^{0}$ is $\mathfrak{J}^{\sigma, u}$-measurable, obeying the equation $X^{n+1}=\left(X^{n}+\xi_{n}\right)^{+}$, and the ergodicity follows from Remark 2.4 and the fact that $X^{n}$ is compatible with the shift $U$.

Let us now consider the case $\theta \geq 0$ and $\mu>0$. The renovation events $A_{n}$ will be constructed now in two steps. We will first introduce a majorizing SRS $X(n)^{*}$ on the same probability space, which will enable us to obtain simple stationary renovating events $A_{n}^{*}$ with positive probability, and $A_{n}$ will be obtained as some subsets of $A_{n}^{*}$. The SRS $X(n)^{*}$ has the following form:

$$
X(0)^{*}=X(0), \quad X(n+1)^{*}=\max \left(C, X(n)^{*}+\xi_{n}^{*}\right),
$$

where

$$
\xi_{n}^{*}=\prod\left(\lambda \sigma_{n}, u_{n}^{1}\right)-I\left(u_{n}^{2} \leq \frac{\theta+C \mu}{\lambda+\theta+C \mu}\right) .
$$

The sequence $\left\{\xi_{n}^{*}\right\}$ is measurable with respect to $\mathfrak{J}^{\sigma, u}$ and $\xi_{n+1}^{*}=U \xi_{n}^{*}$. From this and Remarks 2.3 and 2.4 it follows that the sequence $\left\{\xi_{n}^{*}\right\}$ is stationary and ergodic. We choose the constant $C$ such that $\mathbf{E} \xi_{n}^{*}<0$ if the condition $\lambda \mathbf{E} \sigma_{1}<1$ holds, where

$$
\mathbf{E}\left(\xi_{n}^{*}\right)=\lambda \mathbf{E} \sigma_{1}-\frac{\theta+C \mu}{\lambda+\theta+C \mu}
$$

It follows that there exist renovation events $A_{n}^{*}=T^{n} A_{0}^{*}, n \geq n_{0}$, where $A_{0}^{*}$ is defined as (3.7) with the sequence $\left\{\xi_{n}^{*}\right\}$, such that $X(n)^{*}=C$ on the set $A_{n}^{*}$ for all $n \geq n_{0}$. Define the sets

$$
B_{0}=\left\{\prod\left(\lambda \sigma_{-k}, u_{-k}^{1}\right)=0, u_{-k}^{2} \leq \frac{\theta+k \mu}{\lambda+\theta+k \mu}, k=1, \ldots, C\right\}, \quad B_{n}=T^{n} B_{0} .
$$

The sets $A_{n}=A_{n-C}^{*} \cap B_{n}$ form a stationary renovating sequence for $X(n)$, since for all $n \geq$ $n_{0}+C$, we have on $A_{n}$, the values $X(n-k) \leq k, k=0,1, \ldots, C$, and in particular, $X(n)=0$. Moreover, $P\left(A_{0}\right)=P\left(A_{-C}^{*}\right) P\left(B_{0} \mid A_{-C}^{*}\right)>0$ (see Altman and Borovkov [2, pages 353$354]$ ). The strong coupling convergence of the process $X(n)$ to a stationary ergodic regime follows from Theorem 2.7.

Remark 3.2. Although the conditions of Theorem 3.1 are sufficient for stability, we can assert that they are necessary, since for SRS of the form $X(n+1)=\left(X(n)+\xi_{n}\right)^{+}$, the 
condition $E \xi_{n}>0$ implies that the process $X(n)$ converges in distribution to an improper limiting sequence, that is, $X(n) \rightarrow \infty$ almost surely, and it can be extended under wide assumptions to the case $E \xi_{n}=0$ (see Borovkov [8, Theorem 1.7]).

\section{Stability of the retrial queue with two types of customers}

Consider now a retrial queue with two types of arriving customers, known as "impatient" and "persistent." If an impatient customer finds the server busy, then he leaves the system. On the other hand, if a persistent customer arrives and finds the server busy, then he may have access to the orbit and waits to be served later according to the versatile retrial policy described above. Assume that the interarrival times $\left\{\tau_{n}^{1}\right\}$ of type 1 (impatient) and $\left\{\tau_{n}^{2}\right\}$ of type 2 (persistent) are sequences of independent and identically distributed random variables with exponential distributions with parameters $\lambda_{1}>0$ and $\lambda_{2}>0$, respectively. The service times $\left\{\sigma_{n}^{1}\right\}$ (for type 1 ) and $\left\{\sigma_{n}^{2}\right\}$ (for type 2) are stationary and ergodic, independent of each other and of $\left\{\tau_{n}^{1}\right\},\left\{\tau_{n}^{2}\right\}$, orbit retrial times and retrial times of each customer in orbit, and $0<\mathbf{E}\left(\sigma_{n}^{j}\right)<\infty, j=1,2$. The sequences $\left\{\tau_{n}^{1}\right\},\left\{\tau_{n}^{2}\right\}$, orbit retrial times and retrial times of each customer in orbit are independent of each other. Let $X(t)$ be the number of customers in orbit at time $t$. Define $s_{n}$ to be the instant when the $(n-$ $1)$ st service time ends. We consider the process $X(n)$ embedded immediately after time $s_{n}$, (i.e., $X(n)=X\left(s_{n}^{+}\right)$). Let $u_{n}^{1}$ and $u_{n}^{2}$ defined as in Section 3, and independent of the sequences $\left\{\sigma_{n}^{1}\right\}$ and $\left\{\sigma_{n}^{2}\right\}$, except that $u^{2}=u_{n}^{2}$ will generate now the type of request of service: impatient customer, external persistent customer or persistent customer from the orbit at the end of the successive service periods. The representation of the process $X(n)$ is

$$
X(n+1)=\left(X(n)+\xi_{n}\right)^{+}
$$

where the driving sequence $\left\{\xi_{n}\right\}$ is now of the form

$$
\begin{aligned}
\xi_{n}= & \prod\left(\lambda_{2} \sigma_{n}^{1}, u_{n}^{1}\right) I\left(u_{n}^{2} \leq \frac{\lambda_{1}}{\lambda_{1}+\lambda_{2}+\theta+X(n) \mu}\right) \\
& +\prod\left(\lambda_{2} \sigma_{n}^{2}, u_{n}^{1}\right) I\left(\frac{\lambda_{1}}{\lambda_{1}+\lambda_{2}+\theta+X(n) \mu}<u_{n}^{2} \leq \frac{\lambda_{1}+\lambda_{2}}{\lambda_{1}+\lambda_{2}+\theta+X(n) \mu}\right) \\
& +\prod\left(\lambda_{2} \sigma_{n}^{2}, u_{n}^{1}\right) I\left(\frac{\lambda_{1}+\lambda_{2}}{\lambda_{1}+\lambda_{2}+\theta+X(n) \mu}<u_{n}^{2} \leq 1\right) \\
& -I\left(\frac{\lambda_{1}+\lambda_{2}}{\lambda_{1}+\lambda_{2}+\theta+X(n) \mu}<u_{n}^{2} \leq 1\right) .
\end{aligned}
$$

THEOREM 4.1. The process $X(n)$ is strong coupling convergent to a unique stationary ergodic regime if one of the following conditions is fulfilled:

(1) $\theta>0, \mu=0$, and $\left(\lambda_{1}+\lambda_{2}+\lambda_{2}\left(\theta \mathbf{E} \sigma^{2}+\lambda_{1} \mathbf{E} \sigma^{1}+\lambda_{2} \mathbf{E} \sigma^{2}\right)\right) /\left(\lambda_{1}+\lambda_{2}+\theta\right)<1$;

(2) $\theta \geq 0, \mu>0$, and $\lambda_{2} \mathrm{E} \sigma^{2}<1$. 
10 Stability of retrial queues with versatile retrial policy

Proof. Consider the first case $\theta>0$ and $\mu=0$. Then the sequence $\left\{\xi_{n}\right\}$ has the form

$$
\begin{aligned}
\xi_{n}= & \prod\left(\lambda_{2} \sigma_{n}^{1}, u_{n}^{1}\right) I\left(u_{n}^{2} \leq \frac{\lambda_{1}}{\lambda_{1}+\lambda_{2}+\theta}\right) \\
& +\prod\left(\lambda_{2} \sigma_{n}^{2}, u_{n}^{1}\right) I\left(\frac{\lambda_{1}}{\lambda_{1}+\lambda_{2}+\theta}<u_{n}^{2} \leq \frac{\lambda_{1}+\lambda_{2}}{\lambda_{1}+\lambda_{2}+\theta}\right) \\
& +\prod\left(\lambda_{2} \sigma_{n}^{2}, u_{n}^{1}\right) I\left(\frac{\lambda_{1}+\lambda_{2}}{\lambda_{1}+\lambda_{2}+\theta}<u_{n}^{2} \leq 1\right)-I\left(\frac{\lambda_{1}+\lambda_{2}}{\lambda_{1}+\lambda_{2}+\theta}<u_{n}^{2} \leq 1\right) .
\end{aligned}
$$

Define the $\sigma$-algebras $\mathfrak{I}_{n}^{\sigma, u}=\sigma\left(\sigma_{k}^{1}, \sigma_{k}^{2}, u_{k}^{1}, u_{k}^{2} ; k \leq n\right)$ and $\mathfrak{J}^{\sigma, u}=\sigma\left(\sigma_{k}^{1}, \sigma_{k}^{2}, u_{k}^{1}, u_{k}^{2} ;-\infty<\right.$ $k<\infty)$. Let $U$ be the measure-preserving shift transformation of $\mathfrak{J}^{\sigma, u}$-measurable random variables generated by $\left\{\sigma_{n}^{1}, \sigma_{n}^{2}, u_{n}^{1}, u_{n}^{2} ;-\infty<n<\infty\right\}$. Since $\xi_{n}$ is generated by $\left\{\sigma_{n}^{1}, \sigma_{n}^{2}\right.$, $\left.u_{n}^{1}, u_{n}^{2}\right\}$, then $\left\{\xi_{n} ;-\infty<n<\infty\right\}$ is compatible with the shift $U$. Hence, from Remarks 2.3 and 2.4, the sequence $\left\{\xi_{n} ;-\infty<n<\infty\right\}$ is stationary and ergodic. We have

$$
\mathbf{E}\left(\xi_{n}\right)=\frac{\lambda_{2}\left(\theta \mathbf{E} \sigma^{2}+\lambda_{1} \mathbf{E} \sigma^{1}+\lambda_{2} \mathbf{E} \sigma^{2}\right)-\theta}{\lambda_{1}+\lambda_{2}+\theta},
$$

so that if

$$
\frac{\lambda_{1}+\lambda_{2}+\lambda_{2}\left(\theta \mathbf{E} \sigma^{2}+\lambda_{1} \mathbf{E} \sigma^{1}+\lambda_{2} \mathbf{E} \sigma^{2}\right)}{\lambda_{1}+\lambda_{2}+\theta}<1,
$$

then $\mathbf{E}\left(\xi_{n}\right)<0$ and the rest of the proof is similar to the first case of Theorem 3.1. For the case $\theta \geq 0$ and $\mu>0$, we construct a majorizing SRS as follows:

$$
X(0)^{*}=X(0), \quad X(n+1)^{*}=\max \left(C, X(n)^{*}+\xi_{n}^{*}\right),
$$

where

$$
\begin{aligned}
\xi_{n}^{*}= & \prod\left(\lambda_{2} \sigma_{n}^{1}, u_{n}^{1}\right) I\left(u_{n}^{2} \leq \frac{\lambda_{1}}{\lambda_{1}+\lambda_{2}+\theta+C \mu}\right) \\
& +\prod\left(\lambda_{2} \sigma_{n}^{2}, u_{n}^{1}\right) I\left(\frac{\lambda_{1}}{\lambda_{1}+\lambda_{2}+\theta+C \mu}<u_{n}^{2} \leq \frac{\lambda_{1}+\lambda_{2}}{\lambda_{1}+\lambda_{2}+\theta+C \mu}\right) \\
& +\prod\left(\lambda_{2} \sigma_{n}^{2}, u_{n}^{1}\right) I\left(\frac{\lambda_{1}+\lambda_{2}}{\lambda_{1}+\lambda_{2}+\theta+C \mu}<u_{n}^{2} \leq 1\right)-I\left(\frac{\lambda_{1}+\lambda_{2}}{\lambda_{1}+\lambda_{2}+\theta+C \mu}<u_{n}^{2} \leq 1\right), \\
\mathbf{E}\left(\xi_{n}^{*}\right)= & \frac{\lambda_{2}\left(\lambda_{1} \mathbf{E} \sigma^{1}+\lambda_{2} \mathbf{E} \sigma^{2}\right)}{\lambda_{1}+\lambda_{2}+\theta+C \mu}+\frac{\theta+C \mu}{\lambda_{1}+\lambda_{2}+\theta+C \mu}\left(\lambda_{2} \mathbf{E} \sigma^{2}-1\right) .
\end{aligned}
$$

The sequence $\left\{\xi_{n}^{*}\right\}$ is measurable with respect to $\mathfrak{J}^{\sigma, u}$ and $\xi_{n+1}^{*}=U \xi_{n}^{*}$. From this and Remarks 2.3 and 2.4, it follows that the sequence $\left\{\xi_{n}^{*}\right\}$ is stationary and ergodic. We choose the constant $C$ such that $\mathbf{E} \xi_{n}^{*}<0$ if the condition $\lambda_{2} \mathbf{E} \sigma^{2}<1$ holds, and the rest of the proof is similar to the second part of that of Theorem 3.1. 


\section{Stability of the retrial queue with breakdowns}

Consider a single server retrial queue with the server subject to breakdowns. Primary customers arrive from the outside according to a Poisson process with rate $\lambda$. We consider the versatile retrial policy for the access from the orbit to the server as described in Section 3. Assume that the server fails at times $\beta_{i}, i=1,2, \ldots$, according to a Poisson process with rate $\alpha$, that is, the server fails after an exponential amount of time with mean $1 / \alpha$. If a breakdown occurs at time $\beta_{i}$, the server takes immediately a repair time $r_{i}, i=1,2, \ldots$. We assume that after a repair time the server is as good as new and the service of a customer is cumulative.

We assume that the service times $\left\{\sigma_{n}\right\}$ and repair times $\left\{r_{i}\right\}$ are stationary and ergodic and independent of each other. The interarrival times, orbit retrial times, retrial times of each customer in orbit, and breakdown times are independent of each other and of the sequences $\left\{\sigma_{n}\right\}$ and $\left\{r_{i}\right\}$. Denote by $r^{(n)}=\left(r_{k}^{(n)}, k=1,2, \ldots\right)$ the sequence of repair times that occur during the service time $\sigma_{n}$. Let $X(t)$ be the number of customers in orbit at time $t$. Define $s_{n}$ to be the instant when the $(n-1)$ st service time ends. We consider the process $X(n)$ embedded immediately after time $s_{n}$, (i.e., $X(n)=X\left(s_{n}^{+}\right)$). Let $u_{n}^{1}, u_{n}^{2}, u_{n}^{3}$, and $u_{i}^{(n)}$ be i.i.d. sequences of random variables distributed uniformly on $[0,1]$, mutually independent and independent of the sequences $\left\{\sigma_{n}\right\}$ and $\left\{r_{i}\right\} . u^{1}=\left\{u_{n}^{1}\right\}$ will generate the arrival process, $u^{2}=\left\{u_{n}^{2}\right\}$ will generate the occurrence of breakdowns, $u_{n}^{3}$ will generate the type of arrival (external or from the orbit) at the end of the successive service periods, and $u_{i}^{(n)}$ will generate the arrival process during the $i$ th repair time that occurs during the $n$th service time, denote by $u^{(n)}=\left(u_{i}^{(n)}, i=1,2, \ldots\right)$. We have the following representation of the process $X(n)$ :

$$
X(n+1)=\left(X(n)+\xi_{n}\right)^{+}
$$

where

$$
\xi_{n}=\prod\left(\lambda \sigma_{n}, u_{n}^{1}\right)+\sum_{i=1}^{\prod\left(\alpha \sigma_{n}, u_{n}^{2}\right)} \prod\left(\lambda r_{i}^{(n)}, u_{i}^{(n)}\right)-I\left(u_{n}^{3} \leq \frac{\theta+X(n) \mu}{\lambda+\theta+X(n) \mu}\right)
$$

is the driving sequence.

THeOREM 5.1. The process $X(n)$ is strong coupling convergent to a unique stationary ergodic regime if one of the following conditions is fulfilled:

(1) $\theta>0, \mu=0$, and $\lambda \mathbf{E} \sigma_{1}\left(1+\alpha \mathbf{E} r_{1}\right)<\theta /(\lambda+\theta)$;

(2) $\theta \geq 0, \mu>0$, and $\lambda \mathbf{E} \sigma_{1}\left(1+\alpha \mathbf{E} r_{1}\right)<1$.

Proof. Note that $\prod\left(\lambda \sigma_{n}, u_{n}^{1}\right)$ and $\prod\left(\lambda r_{i}, u_{i}^{(n)}\right)$ represent the number of arrivals during the $n$th service time $\sigma_{n}$ and the $i$ th repair time $r_{i}$, respectively. $\prod\left(\alpha \sigma_{n}, u_{n}^{2}\right)$ counts the number of breakdowns during the processing of the $n$th service duration $\sigma_{n}$. If $\theta>0$ and $\mu=0$, then the driving sequence (5.2) has the following form:

$$
\xi_{n}=\prod\left(\lambda \sigma_{n}, u_{n}^{1}\right)+\sum_{i=1}^{\prod\left(\alpha \sigma_{n}, u_{n}^{2}\right)} \prod\left(\lambda r_{i}, u_{i}^{(n)}\right)-I\left(u_{n}^{3} \leq \frac{\theta}{\lambda+\theta}\right) .
$$


Define the $\sigma$-algebras $\mathfrak{I}_{n}^{\sigma, u, r}=\sigma\left(\sigma_{k}, r^{(k)}, u^{(k)}, u_{k}^{1}, u_{k}^{2}, u_{k}^{3} ; k \leq n\right)$ and $\mathfrak{J}^{\sigma, u, r}=\sigma\left(\sigma_{k}, r^{(k)}\right.$, $\left.u^{(k)}, u_{k}^{1}, u_{k}^{2}, u_{k}^{3} ;-\infty<k<\infty\right)$. Let $U$ be the measure-preserving shift transformation of $\mathfrak{J}^{\sigma, u, r}$-measurable random variables generated by $\left\{\sigma_{n}, r^{(n)}, \mathfrak{u}^{(n)}, u_{n}^{1}, \mathfrak{u}_{n}^{2}, \mathfrak{u}_{n}^{3} ;-\infty<n<\infty\right\}$, then the sequence $\left\{\xi_{n}\right\}$ is measurable with respect to $\mathfrak{J}^{\sigma, u, r}$, and by Remarks 2.3 and 2.4, $\left\{\xi_{n}\right\}$ is stationary and ergodic. From the Wald's equation and the memoryless property of the Poisson process, we get

$$
\mathbf{E} \xi_{n}=\lambda \mathbf{E} \sigma_{1}+\left(\alpha \mathbf{E} \sigma_{1}\right)\left(\lambda \mathbf{E} r_{1}\right)-\frac{\theta}{\lambda+\theta} .
$$

So if $\lambda \mathbf{E} \sigma_{1}\left(1+\alpha \mathbf{E} r_{1}\right)<\theta /(\lambda+\theta)$, then $\mathbf{E} \xi_{n}<0$, and the rest of the proof of the first condition is similar to that of Theorem 3.1.

We now study the case $\theta \geq 0$ and $\mu>0$. Consider the following majorizing SRS:

$$
X^{*}(0)=X(0), \quad X^{*}(n+1)=\max \left(C, X^{*}(n)+\xi_{n}^{*}\right),
$$

where $C$ is an arbitrary integer and

$$
\xi_{n}^{*}=\prod\left(\lambda \sigma_{n}, u_{n}^{1}\right)+\sum_{i=1}^{\prod\left(\alpha \sigma_{n}, u_{n}^{2}\right)} \prod\left(\lambda r_{i}, u_{i}^{(n)}\right)-I\left(u_{n}^{3} \leq \frac{\theta+C \mu}{\lambda+\theta+C \mu}\right) .
$$

The sequence $\left\{\xi_{n}^{*}\right\}$ is measurable with respect to $\mathfrak{J}^{\sigma, u, r}$. From this and Remarks 2.3 and 2.4 , it follows that the sequence $\left\{\xi_{n}^{*}\right\}$ is stationary and ergodic. If the condition $\lambda \mathbf{E} \sigma_{1}(1+$ $\left.\alpha \mathbf{E} r_{1}\right)<1$ holds, we can easily find a constant $C$ such that $\mathbf{E} \xi_{n}^{*}<0$, where now

$$
\mathbf{E}\left(\xi_{n}^{*}\right)=\lambda \mathbf{E} \sigma_{1}+\left(\alpha \mathbf{E} \sigma_{1}\right)\left(\lambda \mathbf{E} r_{1}\right)-\frac{\theta+C \mu}{\lambda+\theta+C \mu} .
$$

The rest of the proof of the second condition is similar to that of Theorem 3.1.

\section{Stability of the retrial queue with negative customers}

We consider now the stability of a single server retrial queue with two types of arrivals, regular and negative. In retrial queues, a regular arriving customer who finds the server busy joins the orbit, and reapplies for service after a random amount of time; otherwise, if he finds the server idle, he receives service and leaves the system. When a negative arrival occurs in a busy system, it immediately removes one regular customer from the orbit if present. Otherwise, if the server is idle, it has no effect on the system. Regular customers arrive from the outside according to a Poisson process with rate $\lambda$. The access from the orbit to the server follows the versatile retrial policy. We assume that the service times $\left\{\sigma_{n}\right\}$ of regular customers are general stationary ergodic. The negative customers arrive according to a Poisson process with rate $\delta$. The interarrival times of regular customers, interarrival times of negative customers, orbit retrial times and retrial times of each customer in orbit are independent of each other and of the sequence $\left\{\sigma_{n}\right\}$. Let $u_{n}^{1}, u_{n}^{2}$, and $u_{n}^{3}$ be three i.i.d. sequences of random variables distributed uniformly on $[0,1]$, mutually independent and independent of the sequences $\left\{\sigma_{n}\right\} . u^{1}=\left\{u_{n}^{1}\right\}$ will generate the arrival 
process of the regular customers, $u^{2}=\left\{u_{n}^{2}\right\}$ will generate the arrival process of the negative customers, and $u_{n}^{3}$ will generate the type of arrival that gains the server (external or from the orbit) at the end of the successive service periods. Let $X(n)$ be defined as above and it has now the following representation:

$$
X(n+1)=\left(X(n)+\zeta_{n}-\eta_{n}\right)^{+}
$$

where

$$
\begin{gathered}
\zeta_{n}=\prod\left(\lambda \sigma_{n}, u_{n}^{1}\right)-I\left(u_{n}^{3} \leq \frac{\theta+X(n) \mu}{\lambda+\theta+X(n) \mu}\right), \\
\eta_{n}=\prod\left(\delta \sigma_{n}, u_{n}^{2}\right) .
\end{gathered}
$$

Theorem 6.1. The process $X(n)$ is strong coupling convergent to a unique stationary and ergodic regime if one of the following conditions is fulfilled:

(1) $\theta>0, \mu=0$, and $((\lambda-\delta)(\lambda+\theta) / \theta) \mathbf{E} \sigma_{1}<1$;

(2) $\theta \geq 0, \mu>0$, and $(\lambda-\delta) \mathbf{E} \sigma_{1}<1$.

Proof. Consider the first case $\theta>0$ and $\mu=0$. Then the sequence (6.2) has the form

$$
\zeta_{n}=\prod\left(\lambda \sigma_{n}, u_{n}^{1}\right)-I\left(u_{n}^{3} \leq \frac{\theta}{\lambda+\theta}\right) .
$$

Define the $\sigma$-algebras $\mathfrak{J}_{n}^{\sigma, u}=\sigma\left(\sigma_{k}, u_{k}^{1}, u_{k}^{2}, u_{k}^{3} ; k \leq n\right)$ and $\mathfrak{J}^{\sigma, u}=\sigma\left(\sigma_{k}, u_{k}^{1}, u_{k}^{2}, u_{k}^{3} ;-\infty<k<\right.$ $\infty)$. Let $U$ be the measure-preserving shift transformation of $\mathfrak{J}^{\sigma, u}$-measurable random variables generated by $\left\{\sigma_{n}, u_{n}^{1}, u_{n}^{2}, u_{n}^{3} ;-\infty<n<\infty\right\}$. Since the sequence $\zeta_{n}$ is generated by $\left\{\sigma_{n}, u_{n}^{1}, u_{n}^{3}\right\}$ and $\eta_{n}$ is generated by $\left\{\sigma_{n}, u_{n}^{2}\right\}$, then by Remarks 2.3 and $2.4, \zeta_{n}$ and $\eta_{n}$ are stationary and ergodic. $\mathbf{E}\left(\zeta_{n}-\eta_{n}\right)=\lambda \mathbf{E} \sigma_{1}-\theta /(\lambda+\theta)-\delta \mathbf{E} \sigma_{1}$ and if

$$
\frac{(\lambda-\delta)(\lambda+\theta)}{\theta} \mathbf{E} \sigma_{1}<1
$$

then we have $\mathbf{E}\left(\zeta_{n}-\eta_{n}\right)<0$, and the rest of the proof is similar to the first part of Theorem 3.1 .

Let us study now the case $\theta \geq 0$ and $\mu>0$. We follow the same method of the second parts of the above theorems by constructing a majorizing SRS $X^{*}(n)$ defined as follows:

$$
X^{*}(0)=X(0), \quad X^{*}(n+1)=\max \left(C, X^{*}(n)+\zeta_{n}^{*}-\eta_{n}\right)
$$

where

$$
\zeta_{n}^{*}=\prod\left(\lambda \sigma_{n}, u_{n}^{1}\right)-I\left(u_{n}^{3} \leq \frac{\theta+C \mu}{\lambda+\theta+C \mu}\right) .
$$

The sequence $\left\{\zeta_{n}^{*}\right\}$ is measurable with respect to $\mathfrak{J}^{\sigma, u}$. From this and Remarks 2.3 and 2.4, it follows that the sequence $\left\{\zeta_{n}^{*}\right\}$ is stationary and ergodic. If $(\lambda-\delta) \mathbf{E} \sigma_{1}<1$, we can easily find a constant $C$ such that $\mathbf{E}\left(\zeta_{n}^{*}-\eta_{n}\right)=(\lambda-\delta) \mathbf{E} \sigma_{1}-(\theta+C \mu) /(\lambda+\theta+C \mu)<0$. The rest of the proof is similar to the second part of Theorem 3.1. 
14 Stability of retrial queues with versatile retrial policy

\section{Stability of the retrial queue with batch arrivals}

Consider a single server retrial queue with the versatile retrial policy. Let us now consider that at every arrival epoch $t_{k}, k=1,2, \ldots$, a random batch of $a_{k}$ customers enters the system. If the server is busy at the arrival epoch, then the whole batch of customers joins the orbit, whereas if the server is free, then one of the arriving customers starts his service and the others join the orbit. We assume that the input flow of customers occurs according to a Poisson process with rate $\lambda$, the sequence of batch sizes $\left\{a_{k}\right\}$ is independent and identically distributed with general distribution and mean $\bar{a}$, where $0<\bar{a}<\infty$. The $n$th service time of a customer is $\sigma_{n}$, where $0<\mathrm{E} \sigma_{n}<\infty$, and we assume that the sequence $\left\{\sigma_{n}\right\}$ is stationary and ergodic. We denote by $a^{(n)}=\left(a_{k}^{(n)}, k=1,2, \ldots\right)$ the size of batches of the arrivals that occur during the service time $\sigma_{n}$. We assume that the input flow of customers, size of batches, orbit retrial times, retrial times of each customer in orbit and service times are mutually independent.

Let $X(n)$ be defined as above and it has the following representation as an SRS:

$$
X(n+1)=\left(X(n)+\xi_{n}\right)^{+}
$$

where

$$
\xi_{n}=\sum_{k=1}^{\prod\left(\lambda \sigma_{n}, u_{n}^{1}\right)} a_{k}^{(n)}+\left(a_{1}-1\right) I\left(u_{n}^{2} \leq \frac{\lambda}{\lambda+\theta+X(n) \mu}\right)-I\left(\frac{\lambda}{\lambda+\theta+X(n) \mu}<u_{n}^{2} \leq 1\right),
$$

and $a_{1}$ is the size batch of the first arrival that gains the server if the arrival occurs before a repeated attempt.

TheOREM 7.1. The process $X(n)$ is strong coupling convergent to a unique stationary and ergodic regime if one of the following conditions is fulfilled:

(1) $\theta>0, \mu=0, \lambda(\bar{a}-1)<\theta$, and $\lambda \bar{a} \mathbf{E} \sigma_{1}<(\lambda(1-\bar{a})+\theta) /(\lambda+\theta)$;

(2) $\theta \geq 0, \mu>0$, and $\lambda \bar{a} \mathrm{E} \sigma_{1}<1$.

Proof. Consider the first case $\theta>0$ and $\mu=0$, so the driving sequence (7.2) will have the following form:

$$
\xi_{n}=\sum_{k=1}^{\Pi\left(\lambda \sigma_{n}, u_{n}^{1}\right)} a_{k}^{(n)}+\left(a_{1}-1\right) I\left(u_{n}^{2} \leq \frac{\lambda}{\lambda+\theta}\right)-I\left(\frac{\lambda}{\lambda+\theta}<u_{n}^{2} \leq 1\right) .
$$

Define the $\sigma$-algebras $\mathfrak{I}_{n}^{\sigma, u, a}=\sigma\left(\sigma_{k}, u_{k}^{1}, u_{k}^{2}, a^{(k)} ; k \leq n\right)$ and $\mathfrak{I}^{\sigma, u, a}=\sigma\left(\sigma_{k}, u_{k}^{1}, u_{k}^{2}, a^{(k)} ;-\infty<\right.$ $k<\infty)$ and we assume that $a_{1}$ is measurable with respect to $\mathfrak{I}_{n}^{\sigma, u, a}$ and $\mathfrak{J}^{\sigma, u, a}$. Let $U$ be the measure-preserving shift transformation of $\mathfrak{J}^{\sigma, u, a}$-measurable random variables generated by $\left\{\sigma_{n}, u_{n}^{1}, u_{n}^{2}, a^{(n)} ;-\infty<n<\infty\right\}$. The sequence $\left\{\xi_{n}\right\}$ is $\mathfrak{I}^{\sigma, u, a}$-measurable, then by Remarks 2.3 and $2.4,\left\{\xi_{n}\right\}$ is stationary and ergodic. We have

$$
\mathbf{E} \xi_{n}=\lambda \bar{a} \mathbf{E} \sigma_{1}+(\bar{a}-1) \frac{\lambda}{\lambda+\theta}-\frac{\theta}{\lambda+\theta}=\lambda \bar{a} \mathbf{E} \sigma_{1}+\bar{a} \frac{\lambda}{\lambda+\theta}-1,
$$


and if the condition $\lambda \bar{a} \mathbf{E} \sigma_{1}<(\lambda(1-\bar{a})+\theta) /(\lambda+\theta)$ holds, then $\mathbf{E} \xi_{n}<0$. The condition $\lambda(\bar{a}-1)<\theta$ follows from the fact that $\lambda(1-\bar{a})+\theta$ must be positive. The rest of the proof is the same as the first part of Theorem 3.1.

Consider now the second part when $\theta \geq 0$ and $\mu>0$. The majorizing SRS has now the form

$$
X^{*}(0)=X(0), \quad X^{*}(n+1)=\max \left(C, X^{*}(n)+\xi_{n}^{*}\right)
$$

where

$$
\xi_{n}^{*}=\sum_{k=1}^{\Pi\left(\lambda \sigma_{n}, u_{n}^{1}\right)} a_{k}^{(n)}+\left(a_{1}-1\right) I\left(u_{n}^{2} \leq \frac{\lambda}{\lambda+\theta+C \mu}\right)-I\left(\frac{\lambda}{\lambda+\theta+C \mu}<u_{n}^{2} \leq 1\right) .
$$

The sequence $\left\{\xi_{n}^{*}\right\}$ is measurable with respect to $\mathfrak{J}^{\sigma, u, a}$. From this and Remarks 2.3 and 2.4 , it follows that the sequence $\left\{\xi_{n}^{*}\right\}$ is stationary and ergodic. We have $\mathbf{E}\left(\xi_{n}^{*}\right)=\lambda \bar{a} \mathbf{E} \sigma_{1}+$ $\bar{a}(\lambda /(\lambda+\theta+C \mu))-1$, so if the condition $\lambda \bar{a} \mathbf{E} \sigma_{1}<1$ holds, we can choose the constant $C$ as large as possible to have $\mathbf{E}\left(\xi_{n}^{*}\right)<0$. The rest of the proof is similar to the second part of Theorem 3.1.

\section{Acknowledgments}

The authors are grateful to the anonymous referee and associate editor for the helpful suggestions and remarks which improved the paper in its present form. The research of the authors has partly been supported by the CNEPRU project Grant no. B1602/02/2003.

\section{References}

[1] I. Akhmarov and N. P. Leont'eva, Conditions for convergence to limit processes, and the strong law of large numbers for queueing systems, Teoriya Veroyatnostei i ee Primeneniya 21 (1976), no. 3, 559-570 (Russian).

[2] E. Altman and A. A. Borovkov, On the stability of retrial queues, Queueing Systems 26 (1997), no. 3-4, 343-363.

[3] J. R. Artalejo, G-networks: a versatile approach for work removal in queueing networks. G-networks, European Journal of Operational Research 126 (2000), no. 2, 233-249.

[4] J. R. Artalejo and A. Gomez-Corral, Steady state solution of a single-server queue with linear repeated requests, Journal of Applied Probability 34 (1997), no. 1, 223-233.

[5] __ Generalized birth and death processes with applications to queues with repeated attempts and negative arrivals, OR Spektrum 20 (1998), no. 1, 5-14.

[6] __ On a single server queue with negative arrivals and request repeated, Journal of Applied Probability 36 (1999), no. 3, 907-918.

[7] S. Asmussen and S. G. Foss, Renovation, regeneration, and coupling in multiple-server queues in continuous time, Frontiers in Pure and Appl. Probab (H. Niemi, et al., eds.), vol. 1, VSP, Utrecht, 1993, pp. 1-6.

[8] A. A. Borovkov, Stochastic Processes in Queueing Theory, Applications of Mathematics, no. 4, Springer, New York, 1976.

[9] , Asymptotic Methods in Queueing Theory, Wiley Series in Probability and Mathematical Statistics: Applied Probability and Statistics, John Wiley \& Sons, Chichester, 1984.

[10] _ Ergodicity and Stability of Stochastic Processes, Wiley Series in Probability and Statistics: Probability and Statistics, John Wiley \& Sons, Chichester, 1998. 


\section{Stability of retrial queues with versatile retrial policy}

[11] J. L. Doob, Stochastic Processes, John Wiley \& Sons, New York; Chapman \& Hall, London, 1953.

[12] G. I. Falin, Aggregate arrival of customers in one-line system with repeated calls, Ukranian Mathematical Journal 28 (1976), no. 4, 437-440.

[13] _ Functioning under nonsteady conditions of a single-channel system with group arrival of requests and repeated calls, Ukrainian Mathematical Journal 33 (1981), no. 4, 429-432.

[14] G. Fayolle, A simple telephone exchange with delayed feedbacks, Teletraffic Analysis and Computer Performance Evaluation (O. J. Boxma, J. W. Cohen, and H. C. Tijms, eds.), Elsevier Science, Amsterdam, 1986, pp. 245-253.

[15] S. G. Foss, The method of renovating events and its applications in queueing theory, Semi-Markov Models (Brussels, 1984), Plenum, New York, 1986, pp. 337-350.

[16] S. G. Foss and V. V. Kalashnikov, Regeneration and renovation in queues, Queueing Systems 8 (1991), no. 3, 211-223.

[17] E. Gelenbe, Product-form queueing networks with negative and positive customers, Journal of Applied Probability 28 (1991), no. 3, 656-663.

[18] E. Gelenbe, P. Glynn, and K. Sigman, Queues with negative arrivals, Journal of Applied Probability 28 (1991), no. 1, 245-250.

[19] Y. Kifer, Ergodic Theory of Random Transformations, Birkhäuser Boston, Massachusetts, 1996.

[20] H. M. Liang and V. G. Kulkarni, Stability condition for a single-server retrial queue, Advances in Applied Probability 25 (1993), no. 3, 690-701.

[21] M. Martin and J. R. Artalejo, Analysis of an $M / G / 1$ queue with two types of impatient units, Advances in Applied Probability 27 (1995), no. 3, 840-861.

Tewfik Kernane: Department of Probability and Statistics, Faculty of Mathematics, University of Sciences and Technology Houary Boumediene, USTHB, BP 32 El-Alia, Algiers, Algeria

E-mail address: tkernane@usthb.dz

Amar Aïssani: Faculty of Electronics and Computer Science, University of Sciences and Technology Houary Boumediene, USTHB, BP 32 El-Alia, 16111 Algiers, Algeria

E-mail address: amraissani@yahoo.fr 


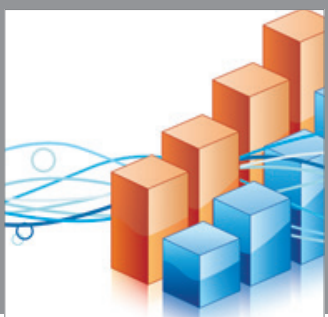

Advances in

Operations Research

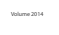

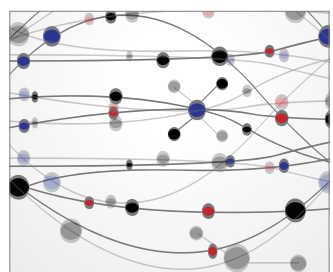

\section{The Scientific} World Journal
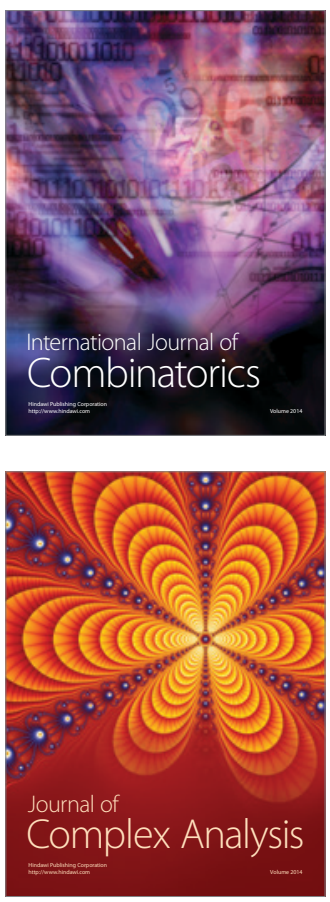

International Journal of

Mathematics and

Mathematical

Sciences
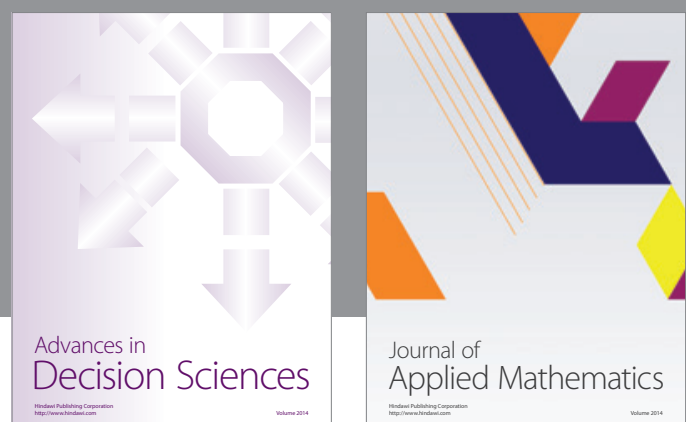

Journal of

Applied Mathematics
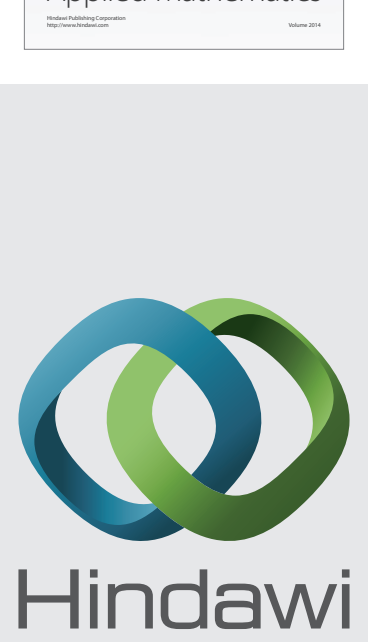

Submit your manuscripts at http://www.hindawi.com
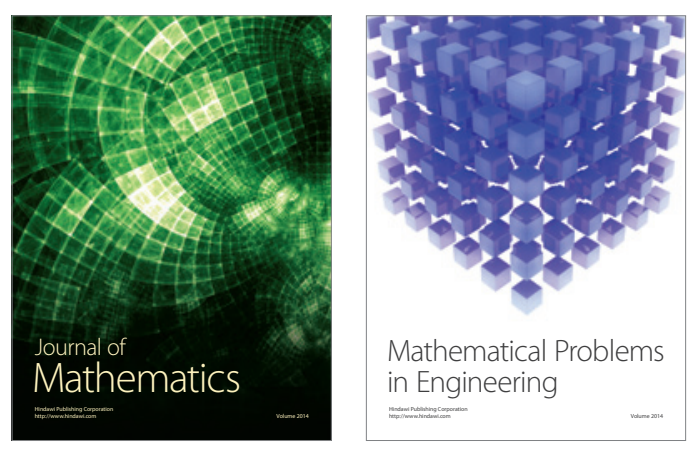

Mathematical Problems in Engineering
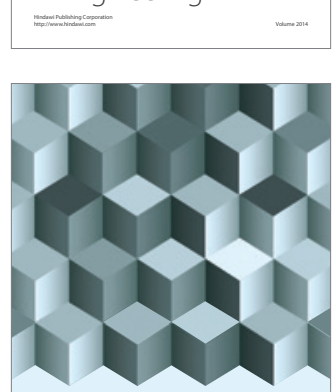

Journal of

Function Spaces
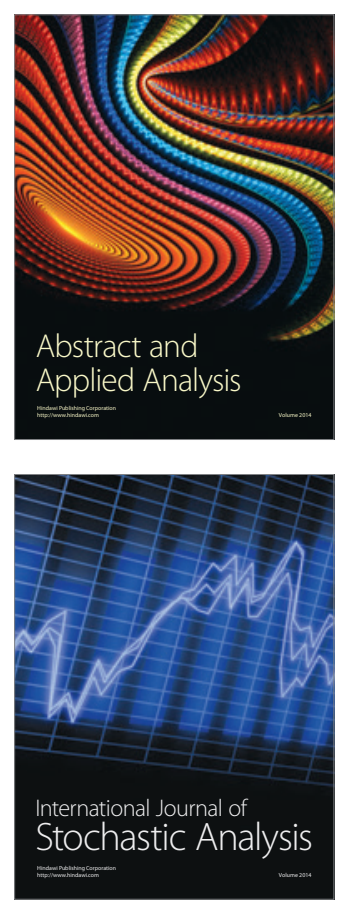

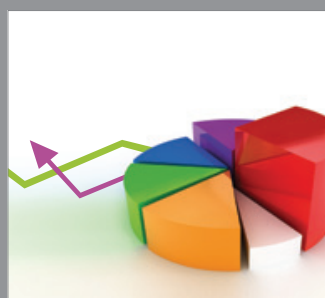

ournal of

Probability and Statistics

Promensencen
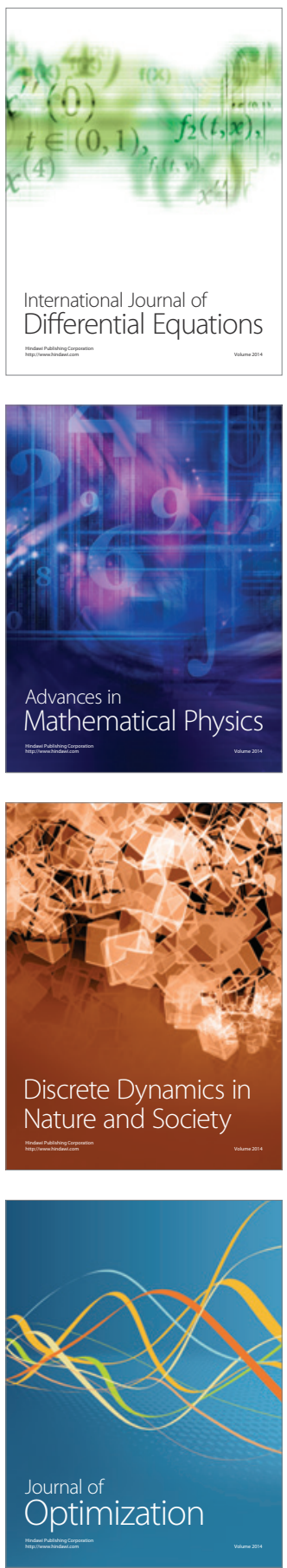